\title{
Communication
}

\section{"Flash" Solvent-free Synthesis of Triazoles Using a Supported Catalyst}

\section{Ibtissem Jlalia $^{1,2}$, Faouzi Meganem ${ }^{2}$, Jean Herscovici ${ }^{1}$ and Christian Girard ${ }^{1, *}$}

1 Laboratoire de Pharmacologie Chimique et Génétique UMR8151 CNRS - U640 INSERM IFR2769, Ecole Nationale Supérieure de Chimie de Paris, 11, rue Pierre et Marie Curie, 75005 Paris, France; E-mails: ibtissemj@yahoo.fr (I. J.), jean-herscovici@enscp.fr (J. H.)

2 Laboratoire de Synthèse Organique et Application, Faculté des Sciences de Bizerte, Université du 7 Novembre à Carthage, 7021 Jarzouna Bizerte, Tunisia; E-mail: Faouzi.Meganem@fss.rnu.tn (F. M.)

* Author to whom correspondence should be addressed; E-mail: christian-girard@enscp.fr.

Received: 8 January 2009; in revised form: 20 January 2009 / Accepted: 22 January 2009 / Published: 22 January 2009

\begin{abstract}
A solvent-free synthesis of 1,4-disubstituted-1,2,3-triazoles using neat azides and alkynes and a copper(I) polymer supported catalyst (Amberlyst $\left.{ }^{\circledR} \mathrm{A} 21 \cdot \mathrm{CuI}\right)$ is presented herein. As it provides the products in high yields and purities within minutes, this method thus being characterized as a "flash" synthesis, and was exemplified through the synthesis of a 24-compound library on a small scale.
\end{abstract}

Keywords: Click chemistry; Huisgen's cycloaddition; Copper (I) catalysis; Triazoles; Supported catalyst; Solvent-free.

\section{Introduction}

Triazoles have gained in interest over the past few years following the introduction of the "clickchemistry" concept [1-3]. This approach concentrates on chemical reactions between highly reactive partners to provide ready access to structures that can be easily diversified, thanks to the generality of those reactions and their relative insensitivity to stereochemical and electronic considerations.

Huisgen's thermal cycloaddition of azides and alkynes to give triazoles [4], was found to be catalyzed by copper(I) (Figure 1) [5-10]. These conditions illustrate the "click" concept perfectly. This 
facilitated the reaction at lower temperature and furthermore only the 1,4-disubstituted regioisomer of the 1,2,3-triazole was formed. The conditions used to conduct such reactions can be addition of copper (I) salts in organic or aqueous systems often in conjunction with a base [11-13], copper (II) salts/ ascorbic acid system (to generate the copper (I) species in situ) [14-16], copper salts adsorbed on zeolites [17], charcoal [18] or clay [19], copper wire [20-22] and nanoparticles/clusters [23-24].

Figure 1. Huisgen's cycloaddition route to 1,2,3-triazoles and its copper (I) catalyzed version.

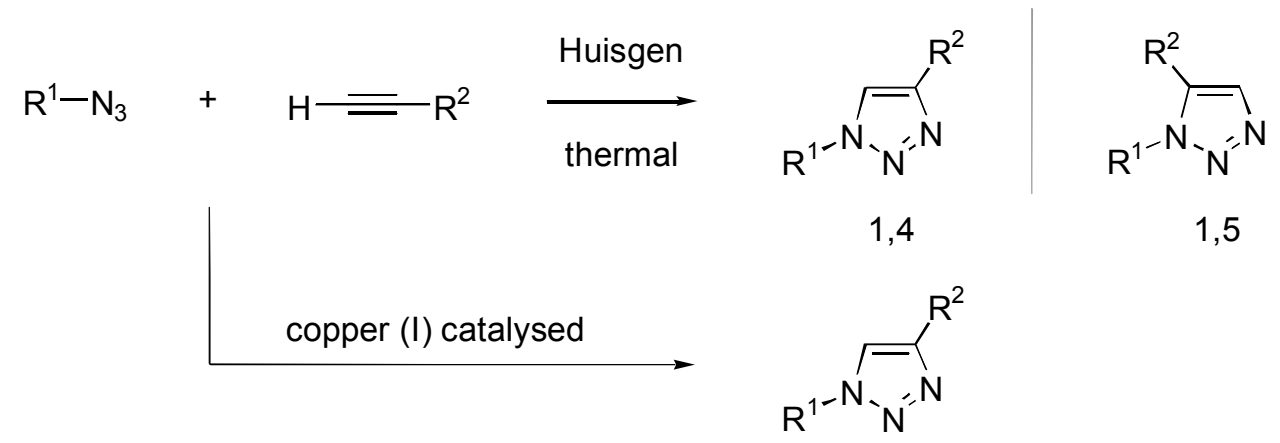

We recently proposed a new catalytic system based on copper (I) iodide chelated on Amberlyst ${ }^{\circledR}$ A21 resin, for use in automated solution synthesis of 1,2,3-triazoles from organic azides and terminal alkynes $[25,26]$. The advantages of this catalyst are the ease of preparation, a good catalytic activity and the simple separation from the reaction product by filtration. This catalyst was successfully employed for the synthesis of triazole libraries in solvents, the reaction needing however a few hours to overnight to be completed.

\section{Results and Discussion}

In recent years there has been a growing pressure on organic chemists to not only find efficient reactions, that can achieve high yields and selectivities, but also to focus on the "greenness" of the processes [27]. One of the major environmental impacts of organic synthesis is the solvent use itself. During our work on this system, we found out that the $\mathrm{A} 21 \cdot \mathrm{CuI}$ polymer was able to catalyze triazole formation within only minutes using neat azides and alkynes derived from various organic structures, i.e. in a solvent-free manner (Scheme 1).

Scheme 1. Solvent-free synthesis of triazoles using a polymer-supported copper (I) iodide.

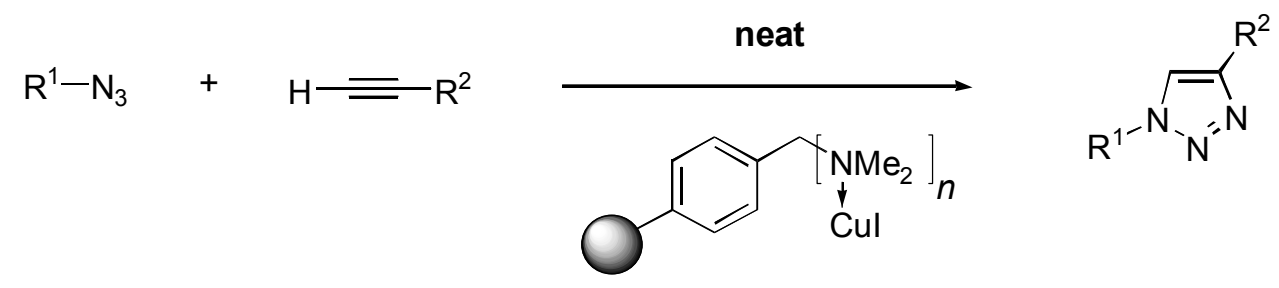

Amberlyst A-21 Cul

We wish to report in this communication a new "flash" (quasi instantaneous) solvent-free method for the synthesis of triazoles using this polymer-supported catalyst [28-31]. When organic azides and 
terminal alkynes were mixed together and treated directly with $\mathrm{A} 21 \cdot \mathrm{CuI}$ catalyst, a rise in the mixture temperature was observed in most cases. As soon as the mixture cooled off, crystallization of the triazole usually occurred within five minutes and this was considered as the end of reaction $(<0.5 \mathrm{~h} v s$. more than $6 \mathrm{~h}$ in solution). Reaction products were then manually separated from the large beads of the catalyst. The results for the synthesis of 24 triazoles are presented in Table 1.

Table 1. Yields for the solvent-free synthesis of a series of triazoles ${ }^{\mathrm{a}}$.

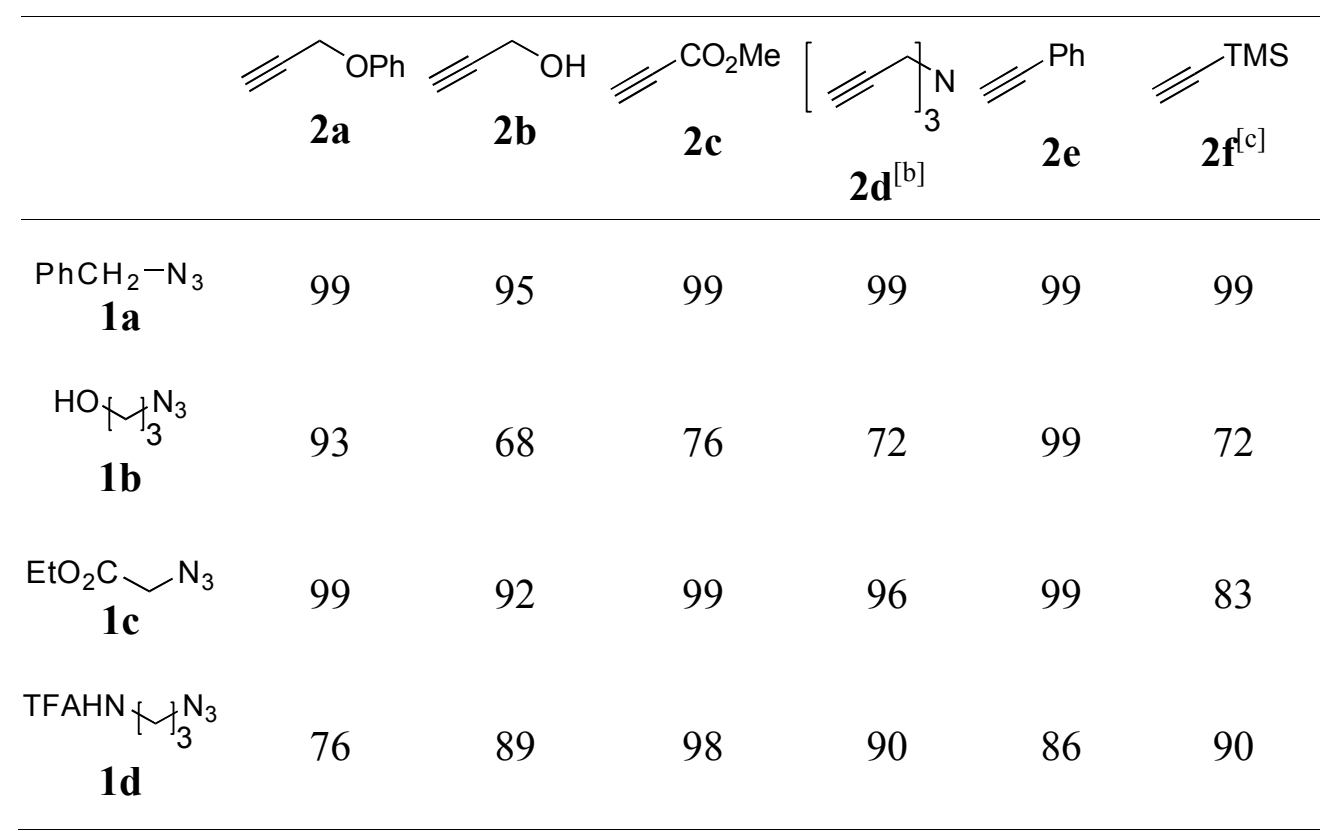

${ }^{a}$ On a $0.5 \mathrm{mmol}$ scale: 1 eq. alkyne/1.1 eq. azide, $30 \mathrm{mg}(8 \% \mathrm{~mol}) \mathrm{A} 21 \cdot \mathrm{CuI}$. All compounds gave correct mp, IR, ${ }^{1} \mathrm{H}-,{ }^{13} \mathrm{C}-\mathrm{NMR}$ and LC-MS analyses; ${ }^{\mathrm{b}}$ with 3.3 eq. azide; ${ }^{\mathrm{c}} 0.75$ eq. azide was used.

In most cases, the yields were high (average 90\%) and for the two-thirds of the formed triazoles were between $90 \%$ and quantitative. In all reactions, only the 1,4-isomer was observed and all products were pure with some minor exceptions. As previously observed with this system, the small excess of azide used was not detected in the reaction products, suggesting a possible sequestration by the polymer.

Yields for the reactions of benzyl azide (1a), ethyl azidoacetate (1c) and 3-azidotrifluoroacetamidopropane (1d) were good (averages between 89-98\%). Products were usually isolated as highly crystalline solids and easily separated from the catalyst. Yields were lower, however, when using 3-azidopropanol (1b) and the corresponding triazoles were isolated in $80 \%$ average yield. This was mainly due to the sticky nature of the products which coated the polymer beads. In this case the reaction yields can be improved by washing the polymer beads with a solvent, but this step obviously diminishes the "greenness" of the approach.

The reaction yields of tripropargylamine (2d) were good when 3.3 eq. of the azide were used, and led exclusively to the corresponding tris(triazoles) 1a-d2d, with no mono- or bis(triazoles) being observed. In this case, traces of copper leaked out from the catalyst due to the presence of the amine centre in the products. This is obviously one of the limitations of this system. However, residual minute amounts of copper can be quickly and easily removed from solutions of the products using polymer-supported thiourea [32]. 
Finally, trimethylsilylacetylene (2f) was found not to react as well as the other alkynes and leading to a coloration of the polymer beads (light orange to red) suggesting side reactions. In order to obtain better conversions, it was the only alkyne used in excess based onto the azide. Yields were also good here in most cases (average 86\%), but still lower for the cycloaddition with $\mathbf{1 b}$.

\section{Conclusions}

We have presented in this communication our findings concerning a new method for the synthesis of 1,4-disubstituted 1,2,3-triazoles. This method provides an "instantaneous" access to the products when using only neat alkynes, azides and an easily prepared polymer-supported copper (I) catalyst, thus being characterized as a "flash" reaction. This method is one of the fastest and easiest compared to traditional thermal and microwave-assisted procedures [33], or to the copper (I) catalyzed versions of Huisgen's cycloaddition themselves. The triazoles were obtained regioselectively in very good yields and purities in only a few minutes, thus improving the access to these heterocycles. We are convinced this method will find many applications for the synthesis of simple and more complex triazole containing molecules.

\section{Experimental}

\section{General}

Chemicals: Copper (I) iodide and propiolic acid methyl ester were purchased from Lancaster. Propargyl alcohol, tripropargylamine and trimethylsilylacetylene were purchased from Aldrich and phenylacetylene from Fluka. These chemicals were used without purification. Propargyl phenyl ether was prepared from propargyl bromide and phenol [34]. Azides were prepared from sodium azide and benzyl bromide, 3-chloropropanol, ethyl bromoacetate and 3-bromopropylamine hydrobromide (after treatment with ethyl trifluoroacetate) following published procedures [35-38]. Solvents: Acetonitrile (spectrometric grade, low water) was purchased from SDS France and used as such. Dichloromethane (SDS France) was treated with phosphorus pentaoxide at reflux $(1 \mathrm{~h})$ before being distilled. Melting points $(\mathrm{mp})$ were determined using a Kofler apparatus after a first evaluation, calibration with a reference sample of a mp near the observed fusion and final measure of the melting point. Infrared spectra were recorded neat on a Jasco FT/IR-4100 in ATR mode (PIKE-MIRacle) between 4,000 and $400 \mathrm{~cm}^{-1}$ and are given in $v\left(\mathrm{~cm}^{-1}\right)$. NMR spectra were recorded on a Bruker Avance DRX instrument in deuteriochloroform (unless otherwise noted) at $300 \mathrm{MHz}$ for the ${ }^{1} \mathrm{H}$ and $75.5 \mathrm{MHz}$ for the ${ }^{13} \mathrm{C}$ spectra. Chemical shifts $(\delta)$ are reported in part per million $(\mathrm{ppm})$ relative to the tetramethylsilane signal as an internal reference. Couplings constants $(J)$ are in hertz and signal multiplicities indicated as s (singlet), d (doublet), $t$ (triplet), q (quadruplet), $m$ (multiplet), dd (doublet of doublets). LC-MS analyses were done on a Shimadzu LCSM-2010 A instrument equipped with a SPD-M10 A PDA diode array detector ( $\mathrm{D}_{2}$, lamp from 190 to $400 \mathrm{~nm}$ ) and an ELSD-LT light scattering detector on an Alltima HP C8 $3 \mu$ (Alltech) reversed phase ( $\mathrm{L}=53 \mathrm{~mm}$; ID = $7 \mathrm{~mm}$ ) HPLC column. The LC were ran using a $1 \mathrm{~mL} / \mathrm{min}$ flow using a gradient between acetonitrile and water containing formic acid $(0,1 \%)$ : 0 to $1 \mathrm{~min}$ : $30 \% \mathrm{CH}_{3} \mathrm{CN}, 1$ to $5 \mathrm{~min}$ : from $30 \%$ to $100 \% \mathrm{CH}_{3} \mathrm{CN}, 5$ to $12 \mathrm{~min}: 100 \% \mathrm{CH}_{3} \mathrm{CN}, 12$ to 14,99 min: from $100 \%$ to $30 \% \mathrm{CH}_{3} \mathrm{CN}, 14,99$ to $20 \mathrm{~min}$ : $30 \% \mathrm{CH}_{3} \mathrm{CN}$. MS was recorded between $\mathrm{m} / \mathrm{z}$ 
$=100$ to 500 at the exit of the column using an ESI ionization and positive ion mode (detector $=1.5$ $\mathrm{kV}$, quadripole $=5 \mathrm{~V}$ ).

\section{Procedures}

Dry Amberlyst ${ }^{\circledR}$ A-21: Commercial wet Amberlyst ${ }^{\circledR}$ A21 resin (Aldrich, 20-50 mesh, $100 \mathrm{~g}$ ) was suspended in $\mathrm{MeOH}(500 \mathrm{~mL})$ for $0.5 \mathrm{~h}$ and filtered (3 times) and then soaked in methylene chloride $(500 \mathrm{~mL})$ for $0.5 \mathrm{~h}$ and again filtered ( 3 times). The resulting resin was placed in a round-bottom flask on a rotoevaporator and dried at $50{ }^{\circ} \mathrm{C}$ under $10 \mathrm{~mm} \mathrm{Hg}$ until it was free-flowing. The dried resin was then kept overnight under vacuum in a desiccator over $\mathrm{P}_{2} \mathrm{O}_{5}$. Specifications from the manufacturer indicate that the polymer contains 4.8 meq. of amine/g of dry resin.

Preparation of the supported catalyst (A-21.CuI): Dry Amberlyst ${ }^{\circledR}$ A21 (1.0 g, 4.8 mmol amine) was added to a solution of copper (I) iodide $(381 \mathrm{mg}, 2.00 \mathrm{mmol})$ in acetonitrile $(15 \mathrm{ml})$ and gently shaken on an orbital stirrer for $17 \mathrm{~h}$. The solvent was drawn off and the resin washed with $\mathrm{CH}_{3} \mathrm{CN}(2 \times 15 \mathrm{~mL})$, $\mathrm{CH}_{2} \mathrm{Cl}_{2}(2 \times 15 \mathrm{~mL})$ and dried under vacuum $(0.01 \mathrm{~mm} \mathrm{Hg})$ at $40^{\circ} \mathrm{C}$. The weight increase was $0.307 \mathrm{~g}$

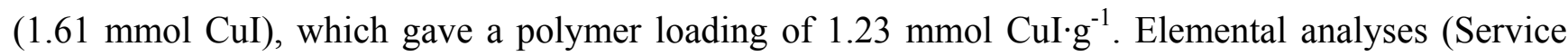
Central d'Analyses du CNRS, Solaize, France) gave a copper content of $8.64 \%$, indicative of a loading

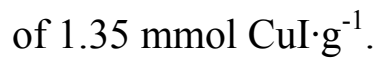

\section{General procedure for triazole synthesis}

CAUTION! Organic azides are potentially explosive and should be handle with care. Even if no incident occurred in this solvent-free reaction on this scale, the cycloaddition can be highly exothermic and should not be attempted on a larger scale, without being aware of explosion risks.

The azide $(0,55 \mathrm{mmol})$ and alkyne $(0,50 \mathrm{mmol})$ were placed together in an open small test tube. Amberlyst $^{\circledR} \mathrm{A} 21 \cdot \mathrm{CuI}(1.35 \mathrm{mmol} / \mathrm{g}, 30 \mathrm{mg}, 0.040 \mathrm{mmol}, 8 \% \mathrm{~mol})$ was added at once. A quick temperature rise was observed in most cases and the triazole crystallized out generally within 5 minutes. After half an hour, which was selected arbitrarily, the product was separated from the catalyst either manually or by dissolution in $\mathrm{CH}_{2} \mathrm{Cl}_{2}$ or $\mathrm{CH}_{3} \mathrm{CN}(3 \times 1 \mathrm{~mL})$ and recovered after evaporation of the solvent.

1-Benzyl-4-(phenoxymethyl)triazole (1a2a): Prepared from $66 \mathrm{mg}(0.50 \mathrm{mmol})$ propargyl phenyl ether and $73 \mathrm{mg}(0.55 \mathrm{mmol})$ benzyl azide. The product was obtained as a white solid (131 $\mathrm{mg}, 99 \%)$. $\mathrm{C}_{16} \mathrm{H}_{15} \mathrm{~N}_{3} \mathrm{O}, \mathrm{M}=265.31 \mathrm{~g} \cdot \mathrm{mol}^{-1}$; mp: 119-121 ${ }^{\circ} \mathrm{C}$; FTIR: v3132, 3016, 2970, 2920, 2866, 1588, 1488, 1239, 1222 and $1052 \mathrm{~cm}^{-1}$; ${ }^{1} \mathrm{H}-\mathrm{NMR}: \delta 5.17(\mathrm{~s}, 2 \mathrm{H}), 5.51(\mathrm{~s}, 2 \mathrm{H}), 6.95(\mathrm{~m}, 3 \mathrm{H}), 7.26(\mathrm{~m}, 4 \mathrm{H}), 7.33$ $(\mathrm{m}, 3 \mathrm{H})$ and $7.44(\mathrm{~s}, 1 \mathrm{H}) \mathrm{ppm} ;{ }^{13} \mathrm{C}-\mathrm{NMR}: \delta 54.2,62.0,114.8,121.3,128.1,128.8,129.1,129.5,134.5$, 144.6 and 158.2 ppm; LC-MS: ELSD pur. $92 \%$, UV pur. $100 \% ; \mathrm{R}_{\mathrm{t}}=9.56 \mathrm{~min} ; \mathrm{m} / \mathrm{z}: 266\left([\mathrm{M}+\mathrm{H}]^{+}\right)$.

3-(4-Phenoxytriazol-1-yl)propan-1-ol (1b2a): Prepared from $66 \mathrm{mg}(0.50 \mathrm{mmol})$ propargyl phenyl ether and $56 \mathrm{mg}(0.55 \mathrm{mmol}) 3$-azidopropanol. The product was obtained as an off-white solid (108 
mg, $93 \%$ ). $\mathrm{C}_{12} \mathrm{H}_{15} \mathrm{~N}_{3} \mathrm{O}_{2}, \mathrm{M}=233.27 \mathrm{~g} \cdot \mathrm{mol}^{-1}$; mp: 39-41 ${ }^{\circ} \mathrm{C}$; FTIR: $v$ 3304, 3132, 3107, 2945, 2870, 1600, 1488, 1239, 1218 and $1052 \mathrm{~cm}^{-1}$; ${ }^{1} \mathrm{H}-\mathrm{NMR}: \delta 2.10$ (q, $\left.J=6.0 \mathrm{~Hz}, 2 \mathrm{H}\right), 3.23(\mathrm{~s}, 1 \mathrm{H}), 3.61(\mathrm{t}, J=6.0$ $\mathrm{Hz}, 2 \mathrm{H}), 4.49(\mathrm{t}, J=6.0 \mathrm{~Hz}, 2 \mathrm{H}), 5.16(\mathrm{~s}, 2 \mathrm{H}), 6.96(\mathrm{~m}, 3 \mathrm{H}), 7.27(\mathrm{~m}, 2 \mathrm{H})$ and $7.66(\mathrm{~s}, 1 \mathrm{H}) \mathrm{ppm} ;{ }^{13} \mathrm{C}-$ NMR: $\delta 32.6,47.1,58.5,61.9,114.7,121.3,123.2,129.5,144.1$ and 158.2 ppm; LC-MS: ELSD pur. $99 \%$, UV pur. $100 \% ; \mathrm{R}_{\mathrm{t}}=4.08 \mathrm{~min} ; \mathrm{m} / z: 234\left([\mathrm{M}+\mathrm{H}]^{+}\right)$.

Ethyl 2-[4-(phenoxymethyl)triazol-1-yl]acetate (1c2a): Prepared from $66 \mathrm{mg}$ (0.50 mmol) propargyl phenyl ether and $71 \mathrm{mg}(0.55 \mathrm{mmol})$ ethyl azidoacetate. The product was obtained as a pale yellow oily solid (129 mg, $99 \%$ ). $\mathrm{C}_{13} \mathrm{H}_{15} \mathrm{~N}_{3} \mathrm{O}_{3}, \mathrm{M}=261.28 \mathrm{~g} \cdot \mathrm{mol}^{-1}$; FTIR: $v 3153,2944,2962,2879,1746$, 1596, 1483, 1471, 1401, $1235,1210,1177$ and $1031 \mathrm{~cm}^{-1}$; ${ }^{1} \mathrm{H}-\mathrm{NMR}: \delta 1.27$ (t, $J=7.2 \mathrm{~Hz}, 3 \mathrm{H}$ ), 4.26 (q, $J=7.2 \mathrm{~Hz}, 2 \mathrm{H}), 5.12(\mathrm{~s}, 2 \mathrm{H}), 5.20(\mathrm{~s}, 2 \mathrm{H}, \mathrm{H}-7), 6.95(\mathrm{~m}, 3 \mathrm{H}), 7.27(\mathrm{~m}, 2 \mathrm{H})$ and $7.73(\mathrm{~s}, 1 \mathrm{H}) \mathrm{ppm} ;{ }^{13} \mathrm{C}-$ NMR: $\delta$ 14.0, 50.8, 61.8, 62.4, 114.8, 121.2, 124.3, 129.5, 144.5, 158.2 and 166.2 ppm; LC-MS : ELSD pur. $97 \%$, UV pur. $100 \% ; \mathrm{R}_{\mathrm{t}}=8.66 \mathrm{~min} ; \mathrm{m} / z: 262\left([\mathrm{M}+\mathrm{H}]^{+}\right)$.

2,2,2-Trifluoro-N-[3-(4-phenoxymethyl-[1,2,3]triazol-1-yl)-propyl]-acetamide (1d2a): Prepared from $66 \mathrm{mg}(0.50 \mathrm{mmol})$ propargyl phenyl ether and $108 \mathrm{mg}(0.55 \mathrm{mmol}) \mathrm{N}$-(trifluoracetyl)-1-azido-3aminopropane. The product was obtained as a white solid (126 mg, $76 \%$ ). $\mathrm{C}_{14} \mathrm{H}_{15} \mathrm{~F}_{3} \mathrm{~N}_{4} \mathrm{O}_{2}, \mathrm{M}=328.30$ $\mathrm{g} \cdot \mathrm{mol}^{-1}$. mp: 78-80 ${ }^{\circ} \mathrm{C}$; FTIR: $v 3356,3140,3102,2958,2883,1704,1600,1559,1488,1243,1206$ and $1168 \mathrm{~cm}^{-1}$; ${ }^{1} \mathrm{H}-\mathrm{NMR}\left(\mathrm{CD}_{3} \mathrm{CN}\right): \delta 2.16(\mathrm{q}, J=6.92 \mathrm{~Hz}, 2 \mathrm{H}), 3.32(\mathrm{t}, J=6.5 \mathrm{~Hz}, 2 \mathrm{H}), 4.42(\mathrm{t}, J=6.5 \mathrm{~Hz}$, $2 \mathrm{H}), 5.17(\mathrm{~s}, 2 \mathrm{H}), 6.97(\mathrm{~m}, 3 \mathrm{H}) 7.28(\mathrm{~m}, 2 \mathrm{H}), 7.80(\mathrm{~s}, 1 \mathrm{H})$ and $7.90(\mathrm{~s}, 1 \mathrm{H}) \mathrm{ppm} ;{ }^{13} \mathrm{C}-\mathrm{NMR}\left(\mathrm{CD}_{3} \mathrm{CN}\right): \delta$ 29.1, 37.0, 47.7, 61.7, 114.7, 117.7, 121.4, 123.3, 129.6, 144.5, 157.6 and 158.1 ppm; LC-MS : ELSD pur. $99 \%$, UV pur. $100 \%$; $\mathrm{R}_{\mathrm{t}}=8.37 \mathrm{~min} ; \mathrm{m} / \mathrm{z}: 329\left([\mathrm{M}+\mathrm{H}]^{+}\right)$.

(1-Benzyltriazol-4-yl)methanol (1a2b): Prepared from $28 \mathrm{mg}(0.50 \mathrm{mmol})$ propargyl alcohol and 73 $\mathrm{mg}(0.55 \mathrm{mmol})$ benzyl azide. The product was obtained as white solid (89 mg, $95 \%) \cdot \mathrm{C}_{10} \mathrm{H}_{11} \mathrm{~N}_{3} \mathrm{O}, \mathrm{M}$ $=189.22 \mathrm{~g} \cdot \mathrm{mol}^{-1}$; mp: $76-78^{\circ} \mathrm{C}$; FTIR: $v$ 3257, 3144, 3091, 2953, 2920, 1451, and $1048 \mathrm{~cm}^{-1} ;{ }^{1} \mathrm{H}-$ NMR: $\delta 4.11(\mathrm{~s}, 1 \mathrm{H}), 4.78(\mathrm{~s}, 2 \mathrm{H}), 5.46(\mathrm{~s}, 2 \mathrm{H}), 7.30(\mathrm{~m}, 5 \mathrm{H})$ and $7.91(\mathrm{~s}, 1 \mathrm{H}) \mathrm{ppm} ;{ }^{13} \mathrm{C}-\mathrm{NMR}: \delta 54.1$, 56.0, 122.0, 128.1, 128.7 , 129.1, 134.5 and 148.0 ppm; LC-MS : ELSD pur. 97\%, UV pur. 100\%; $\mathrm{R}_{\mathrm{t}}$ $=3.41 \mathrm{~min} ; \mathrm{m} / \mathrm{z}: 190\left([\mathrm{M}+\mathrm{H}]^{+}\right)$.

3-[4-(Hydroxymethyl)triazol-1-yl]propan-1-ol (1b2b): Prepared from $28 \mathrm{mg}$ (0.50 mmol) propargyl alcohol and $56 \mathrm{mg}(0.55 \mathrm{mmol}) 3$-azidopropanol. The product was obtained as a viscous colorless oil (53 mg, 68\%). $\mathrm{C}_{6} \mathrm{H}_{11} \mathrm{~N}_{3} \mathrm{O}_{2}, \mathrm{M}=157.17 \mathrm{~g} \cdot \mathrm{mol}^{-1}$; FTIR: $v$ 3382, 3142, 2944, 2881, 1658, 1437, 1344, 1219, 1138 and $1056 \mathrm{~cm}^{-1}$; ${ }^{1} \mathrm{H}-\mathrm{NMR}\left(\mathrm{CD}_{3} \mathrm{CN}\right): \delta 2.02$ (q, $\left.J=6.0 \mathrm{~Hz}, 2 \mathrm{H}\right), 3.24(\mathrm{~s}, 1 \mathrm{H}), 3.49(\mathrm{t}, J=6.0$ $\mathrm{Hz}, 2 \mathrm{H}), 4.41(\mathrm{t}, J=6.0 \mathrm{~Hz}, 2 \mathrm{H}), 4.63(\mathrm{~s}, 2 \mathrm{H})$ and $7.66(\mathrm{~s}, 1 \mathrm{H}) \mathrm{ppm} ;{ }^{13} \mathrm{C}-\mathrm{NMR}\left(\mathrm{CD}_{3} \mathrm{CN}\right): \delta 33.8,47.5$, 56.5, 58.8, 122.8 and 148.9 ppm; LC-MS: ELSD pur. $90 \%$, UV pur. $100 \%$; $\mathrm{R}_{\mathrm{t}}=2.67 \mathrm{~min} ; \mathrm{m} / \mathrm{z}: 158$ $\left([\mathrm{M}+\mathrm{H}]^{+}\right)$.

Ethyl 2-[4-(hydroxymethyl)triazol-1-yl]acetate (1c2b): Prepared from $28 \mathrm{mg}$ (0.50 mmol) propargyl alcohol and $71 \mathrm{mg}(0.55 \mathrm{mmol})$ ethyl azidoacetate. The product was obtained as a pale yellow oily solid (95 mg, $92 \%$ ). $\mathrm{C}_{7} \mathrm{H}_{11} \mathrm{~N}_{3} \mathrm{O}_{3}, \mathrm{M}=185.18 \mathrm{~g} \cdot \mathrm{mol}^{-1}$; FTIR: $v 3110,3076,3038,2849,1708,1210$ and $1023 \mathrm{~cm}^{-1}$; ${ }^{1} \mathrm{H}-\mathrm{NMR}$ : $\delta 1.26(\mathrm{t}, J=7.2 \mathrm{~Hz}, 3 \mathrm{H}), 4.21(\mathrm{q}, J=7.2 \mathrm{~Hz}, 2 \mathrm{H}), 4.72(\mathrm{~s}, 2 \mathrm{H}), 5.12(\mathrm{~s}, 2 \mathrm{H})$ 
and $7.67(\mathrm{~s}, 1 \mathrm{H}) \mathrm{ppm} ;{ }^{13} \mathrm{C}-\mathrm{NMR}: \delta$ 14.0, 50.8, 56.1, 62.4, 123.8, 148.3 and 166.5 ppm; LC-MS: ELSD pur. $99 \%$, UV pur. $100 \% ; \mathrm{R}_{\mathrm{t}}=3.02 \mathrm{~min} ; \mathrm{m} / \mathrm{z}: 186\left([\mathrm{M}+\mathrm{H}]^{+}\right), 208([\mathrm{M}+\mathrm{Na}])^{+}$.

2,2,2-Trifluoro-N-[3-(4-hydroxymethyl-[1,2,3]triazol-1-yl)-propyl]-acetamide (1d2b): Prepared from $28 \mathrm{mg}(0.50 \mathrm{mmol})$ propargyl alcohol and $108 \mathrm{mg}(0.55 \mathrm{mmol}) \mathrm{N}$-(trifluoracetyl)-1-azido-3-aminopropane. The product was obtained as a beige solid (112 mg, $89 \%$ ). $\mathrm{C}_{8} \mathrm{H}_{11} \mathrm{~F}_{3} \mathrm{~N}_{4} \mathrm{O}_{2} . \mathrm{M}=252.20 \mathrm{~g} \cdot \mathrm{mol}^{-1}$; mp: 90-92 ${ }^{\circ}$; FTIR: $v 3306,3219,3061,2949,1721,1576,1467,1181$ and $1069 \mathrm{~cm}^{-1}$; ${ }^{1} \mathrm{H}-\mathrm{NMR}$ $\left(\mathrm{CD}_{3} \mathrm{CN}\right): \delta 2.16(\mathrm{q}, J=6.5 \mathrm{~Hz}, 2 \mathrm{H}), 3.40(\mathrm{t}, J=6.5 \mathrm{~Hz}, 2 \mathrm{H}), 4.41(\mathrm{t}, J=6.0 \mathrm{~Hz}, 2 \mathrm{H}), 4.65(\mathrm{~s}, 2 \mathrm{H}), 7.78$ $(\mathrm{s}, 1 \mathrm{H})$ and $7.90(\mathrm{~s}, 1 \mathrm{H}) \mathrm{ppm} ;{ }^{13} \mathrm{C}-\mathrm{NMR}\left(\mathrm{CD}_{3} \mathrm{CN}\right): \delta 28.9,36.6,38.6,47.9,55.0$ and $122.7 \mathrm{ppm}$; LCMS: ELSD pur. $99 \%$, UV pur. $100 \%$; $\mathrm{R}_{\mathrm{t}}=2.07 \mathrm{~min} ; \mathrm{m} / \mathrm{z}: 253\left([\mathrm{M}+\mathrm{H}]^{+}\right)$.

Methyl 1-benzyltriazole-4-carboxylate (1a2c): Prepared from $42 \mathrm{mg}(0.50 \mathrm{mmol})$ propiolic acid methyl ester and $73 \mathrm{mg}(0.55 \mathrm{mmol})$ benzyl azide. The product was obtained as an off-white solid (108 $\mathrm{mg}$, $99 \%) . \mathrm{C}_{11} \mathrm{H}_{11} \mathrm{~N}_{3} \mathrm{O}_{2}, \mathrm{M}=217.23 \mathrm{~g} \cdot \mathrm{mol}^{-1}$; mp: $115-117{ }^{\circ} \mathrm{C}$; FTIR: $v 3112,3066,3038,2849,1725$, 1538, 1239 and $1048 \mathrm{~cm}^{-1}$; ${ }^{1} \mathrm{H}-\mathrm{NMR}: \delta 3.90(\mathrm{~s}, 3 \mathrm{H}), 5.55(\mathrm{~s}, 2 \mathrm{H}), 7.30(\mathrm{~m}, 2 \mathrm{H}), 7.38(\mathrm{~m}, 3 \mathrm{H})$ and 8.02 (s, 1H) ppm; ${ }^{13} \mathrm{C}-\mathrm{NMR}: \delta 52.2,54.5,127.3,128.3,129.2,129.3,133.6,140.3$ and $161.1 \mathrm{ppm}$; LC-MS: ELSD pur. $98 \%$, UV pur. $100 \%$; $\mathrm{R}_{\mathrm{t}}=8.88 \mathrm{~min} ; \mathrm{m} / \mathrm{z}: 218\left([\mathrm{M}+\mathrm{H}]^{+}\right)$.

Methyl 1-(3-hydroxypropyl)triazole-4-carboxylate (1b2c): Prepared from $42 \mathrm{mg}$ (0.50 mmol) propiolic acid methyl ester and $56 \mathrm{mg}(0.55 \mathrm{mmol}) 3$-azidopropanol. The product was obtained as a beige solid (70 mg, $76 \%$ ). $\mathrm{C}_{7} \mathrm{H}_{11} \mathrm{~N}_{3} \mathrm{O}_{3}, \mathrm{M}=185.18 \mathrm{~g} \cdot \mathrm{mol}^{-1}$; mp: 55-57 ${ }^{\circ} \mathrm{C}$; FTIR: v3124, 2959, 2875, 1737, 1721, 1543, 1223 and $1044 \mathrm{~cm}^{-1}$; ${ }^{1} \mathrm{H}-\mathrm{NMR}: \delta 2.18$ (q, $\left.J=6.0 \mathrm{~Hz}, 2 \mathrm{H}\right), 3.67$ (t, $J=6.0 \mathrm{~Hz}, 2 \mathrm{H}$ ), 3.93 (s, 3H), $4.62(\mathrm{t}, J=6.0 \mathrm{~Hz}, 2 \mathrm{H})$ and $8.22(\mathrm{~s}, 1 \mathrm{H}) \mathrm{ppm} ;{ }^{13} \mathrm{C}-\mathrm{NMR}: \delta 32.4,47.5,52.2,58.3,128.2,139.7$ and 161.2 ppm; LC-MS: ELSD pur. $96 \%$, UV pur. $100 \% ; \mathrm{R}_{\mathrm{t}}=2.98 \mathrm{~min} ; \mathrm{m} / \mathrm{z}: 186\left([\mathrm{M}+\mathrm{H}]^{+}\right), 208$ $([\mathrm{M}+\mathrm{Na}])^{+}$.

Methyl 1-(ethoxycarbonylmethyl)triazole-4-carboxylate (1c2c): Prepared from $42 \mathrm{mg}(0.50 \mathrm{mmol})$ propiolic acid methyl ester and $71 \mathrm{mg}(0.55 \mathrm{mmol})$ ethyl azidoacetate. The product was obtained as a beige solid (105 mg, $99 \%$ ). $\mathrm{C}_{8} \mathrm{H}_{11} \mathrm{~N}_{3} \mathrm{O}_{4}, \mathrm{M}=213.19 \mathrm{~g} \cdot \mathrm{mol}^{-1}$; mp: 102-104 ${ }^{\circ} \mathrm{C}$; FTIR: $v$ 3149, 3007, 2968, 1765, 1718, 1543, 1443, 1380, 1219 and $1032 \mathrm{~cm}^{-1}$; ${ }^{1} \mathrm{H}-\mathrm{NMR}: \delta 1.27$ (t, $J=7.2 \mathrm{~Hz}, 3 \mathrm{H}$ ), 3.92 (s, $3 \mathrm{H}), 4.25$ (q, $J=7.2 \mathrm{~Hz}, 2 \mathrm{H}), 5.22(\mathrm{~s}, 2 \mathrm{H})$ and $8.27(\mathrm{~s}, 1 \mathrm{H}) \mathrm{ppm} ;{ }^{13} \mathrm{C}-\mathrm{NMR}: \delta 14.2,50.8,52.4,62.8$ and 129.3 ppm; LC-MS: ELSD pur. $91 \%$, UV pur. $100 \%$; $\mathrm{R}_{\mathrm{t}}=3.68 \mathrm{~min} ; \mathrm{m} / \mathrm{z}: 214\left([\mathrm{M}+\mathrm{H}]^{+}\right)$.

1-[3-(2,2,2-Trifluoro-acetylamino)-propyl]-1H-[1,2,3]triazole-4-carboxylic acid methyl ester (1d2c): Prepared from $42 \mathrm{mg}(0.50 \mathrm{mmol})$ propiolic acid methyl ester and $108 \mathrm{mg}(0.55 \mathrm{mmol}) \mathrm{N}$ (trifluoracetyl)-1-azido-3-aminopropane. The product was obtained as a beige solid (138 $\mathrm{mg}, 98 \%$ ). $\mathrm{C}_{9} \mathrm{H}_{11} \mathrm{~F}_{3} \mathrm{~N}_{4} \mathrm{O}_{3}, \mathrm{M}=280.21 \mathrm{~g} \cdot \mathrm{mol}^{-1}$; mp: 66-68 ${ }^{\circ} \mathrm{C}$; FTIR: $v$ 3290, 3137, 3094, 2962, 1559, 1219, 1166 and $1048 \mathrm{~cm}^{-1}$; ${ }^{1} \mathrm{H}-\mathrm{NMR}\left(\mathrm{CD}_{3} \mathrm{CN}\right): \delta 2.18(\mathrm{q}, J=6.0 \mathrm{~Hz}, 2 \mathrm{H}), 3.37(\mathrm{t}, J=6.0 \mathrm{~Hz}, 2 \mathrm{H}), 4.45(\mathrm{t}, J=6.0$ $\mathrm{Hz}, 2 \mathrm{H}), 4.50(\mathrm{t}, J=6.5,2 \mathrm{H}), 7.86(\mathrm{~s}, 1 \mathrm{H})$ and $8.35(\mathrm{~s}, 1 \mathrm{H}) \mathrm{ppm} ;{ }^{13} \mathrm{C}-\mathrm{NMR}\left(\mathrm{CD}_{3} \mathrm{CN}\right): \delta 28.5,37.6$, 49.1, 50.5, 126.2, $128.1,133.9$ and 161.1 ppm; LC-MS: ELSD pur. $80 \%$, UV pur. $100 \%$; $\mathrm{R}_{\mathrm{t}}=3.32$ $\min ; m / z: 281\left([\mathrm{M}+\mathrm{H}]^{+}\right)$. 
Tris-(4-benzyl-[1,2,3]triazol-1-ylmethyl)-amine (1a2d): Prepared from $69 \mathrm{mg}(0.50 \mathrm{mmol})$ tripropargylamine and $220 \mathrm{mg}(1.65 \mathrm{mmol})$ benzyl azide. The product was obtained as a white solid (262 mg, 99 \%). $\mathrm{C}_{30} \mathrm{H}_{30} \mathrm{~N}_{10}, \mathrm{M}=530.64 \mathrm{~g} \cdot \mathrm{mol}^{-1}$; mp: 146-148 ${ }^{\circ} \mathrm{C}$; FTIR: $v$ 3139, 3098, 3062, 2950, 2934, 1634, 1429, 1332, 1091 and $1050 \mathrm{~cm}^{-1}$; ${ }^{1} \mathrm{H}-\mathrm{NMR}: \delta 3.69(\mathrm{~s}, 6 \mathrm{H}), 5.50(\mathrm{~s}, 6 \mathrm{H}), 7.26(\mathrm{~m}, 6 \mathrm{H})$, $7.34(\mathrm{~m}, 9 \mathrm{H})$ and $7.66(\mathrm{~s}, 3 \mathrm{H}) \mathrm{ppm} ;{ }^{13} \mathrm{C}-\mathrm{NMR}: \delta 47.3,54.2,123.8,128.1,128.7,134.9$ and $144.4 \mathrm{ppm}$; LC-MS: ELSD pur. $96 \%$, UV pur. $100 \% ; \mathrm{R}_{\mathrm{t}}=6.5 \mathrm{~min} ; \mathrm{m} / \mathrm{z}: 531\left([\mathrm{M}+\mathrm{H}]^{+}\right)$.

Tris(4-(3-hydroxy-propoyl)-[1,2,3]triazol-1-ylmethyl)amine (1b2d): Prepared from $69 \mathrm{mg}$ (0.50 mmol) tripropargylamine and $167 \mathrm{mg}(1.65 \mathrm{mmol}) 3$-azidopropanol. The product was obtained as pale green solid (156 mg, $72 \%$ ). $\mathrm{C}_{18} \mathrm{H}_{30} \mathrm{~N}_{10} \mathrm{O}_{3}, \mathrm{M}=434.50 \mathrm{~g} \cdot \mathrm{mol}^{-1}$; mp: 104-106 ${ }^{\circ} \mathrm{C}$; FTIR: v 3400, 3139, 2950, 2919, 1644, 1460, 1329 and $1055 \mathrm{~cm}^{-1}$; ${ }^{1} \mathrm{H}-\mathrm{NMR}: \delta 1.57(\mathrm{~m}, 6 \mathrm{H}), 3.70(\mathrm{~s}, 6 \mathrm{H}), 4.74(\mathrm{~s}, 3 \mathrm{H}), 5.16(\mathrm{t}$, $J=4.5,6 \mathrm{H}), 5.30(\mathrm{~m}, 6 \mathrm{H})$ and $7.87(\mathrm{~s}, 3 \mathrm{H}) \mathrm{ppm} ;{ }^{13} \mathrm{C}-\mathrm{NMR}: \delta 31.3,45.9,48.3,56.8$ and $123.3 \mathrm{ppm}$; LC-MS: ELSD pur. $90 \%$, UV pur. 100\%; $\mathrm{R}_{\mathrm{t}}=2 \mathrm{~min} ; \mathrm{m} / z: 436\left([\mathrm{M}+\mathrm{H}]^{+}\right)$.

Tris((4-ethoxycarbonylmethyl)-[1,2,3]triazol-1-ylmethyl)amine (1c2d): Prepared from $69 \mathrm{mg}(0.50$ mmol) tripropargylamine and $213 \mathrm{mg}(1.65 \mathrm{mmol})$ ethyl azidoacetate. The product was obtained as a beige solid (249 mg, $96 \%$ ). $\mathrm{C}_{21} \mathrm{H}_{30} \mathrm{~N}_{10} \mathrm{O}_{6}, \mathrm{M}=518.53 \mathrm{~g} \cdot \mathrm{mol}^{-1}$; mp: 110-112 ${ }^{\circ} \mathrm{C}$; FTIR: $v 3144,2986$, 2939, 1735, 1639, 1224 and $1045 \mathrm{~cm}^{-1}$; ${ }^{1} \mathrm{H}-\mathrm{NMR}: \delta 1.29$ (t, $\left.J=6.9,9 \mathrm{H}\right), 3.83$ (s, 6H), 4.25 (q, $J=7.2$, $3 \mathrm{H}), 5.50(\mathrm{~s}, 6 \mathrm{H})$ and $7.87(\mathrm{~s}, 3 \mathrm{H}) \mathrm{ppm} ;{ }^{13} \mathrm{C}-\mathrm{NMR}: \delta 14.5,51.0,62.3,110.0$ and $166.3 \mathrm{ppm}$; LC-MS: ELSD pur. $90 \%$, UV pur. $100 \% ; \mathrm{R}_{\mathrm{t}}=3.2 \mathrm{~min} ; \mathrm{m} / \mathrm{z}: 519\left([\mathrm{M}+\mathrm{H}]^{+}\right)$.

Tris[4-(3-(2,2,2,-trifluoro-acetylamino)-propyl)]-[1,2,3]triazol-1-ylmethyl)amine (1d2d): Prepared from $78 \mathrm{mg}(0.50 \mathrm{mmol})$ tripropargylamine and $108 \mathrm{mg}(1.65 \mathrm{mmol}) \mathrm{N}$-(trifluoracetyl)-1-azido-3aminopropane. The product was obtained as a beige oily solid (324 mg, $90 \%$ ). $\mathrm{C}_{21} \mathrm{H}_{30} \mathrm{~F}_{9} \mathrm{~N}_{13} \mathrm{O}_{3}, \mathrm{M}=$ $719.58 \mathrm{~g} \cdot \mathrm{mol}^{-1}$; FTIR: $v 3269,3133,3087,2939,2897,1716,1565,1445,1355,1215,1203,1150$ and $1049 \mathrm{~cm}^{-1} ;{ }^{1} \mathrm{H}-\mathrm{NMR}\left(\mathrm{CD}_{3} \mathrm{CN}\right): \delta 2.16(\mathrm{q}, J=6.5 \mathrm{~Hz}, 2 \mathrm{H}), 3.27(\mathrm{t}, J=6.5 \mathrm{~Hz}, 2 \mathrm{H}), 3.71(\mathrm{~s}, 6 \mathrm{H}), 4.37$ (t, $J=6.0 \mathrm{~Hz}, 2 \mathrm{H}), 7.78(\mathrm{~s}, 3 \mathrm{H})$ and $7.90(\mathrm{~s}, 3 \mathrm{H}) \mathrm{ppm} ;{ }^{13} \mathrm{C}-\mathrm{NMR}: \delta 29.0,36.8,47.2,47.6144 .3$ and 157.8 ppm; LC-MS: ELSD pur. $92 \%$, UV pur. $90 \%$; $\mathrm{R}_{\mathrm{t}}=1.86 \mathrm{~min} ; \mathrm{m} / z: 720\left([\mathrm{M}+\mathrm{H}]^{+}\right)$.

1-Benzyl-4-phenyl-1H-[1,2,3]triazole (1a2e): Prepared from $51 \mathrm{mg}(0.50 \mathrm{mmol})$ phenylacetylene and $73 \mathrm{mg}(0.55 \mathrm{mmol})$ benzyl azide. The product was obtained as a white solid (117 $\mathrm{mg}, 99 \%)$. $\mathrm{C}_{15} \mathrm{H}_{13} \mathrm{~N}_{3}, \mathrm{M}=235.29 \mathrm{~g} \cdot \mathrm{mol}^{-1}$; mp: $126-128{ }^{\circ} \mathrm{C}$; FTIR: $v 3144,3037,2975,1496$ and $1044 \mathrm{~cm}^{-1} ;{ }^{1} \mathrm{H}-$ NMR: $\delta 5.53(\mathrm{~s}, 2 \mathrm{H}), 7.32(\mathrm{~m}, 8 \mathrm{H}), 7.65(\mathrm{~s}, 1 \mathrm{H})$ and $7.77(\mathrm{~m}, 2 \mathrm{H}) \mathrm{ppm} ;{ }^{13} \mathrm{C}-\mathrm{NMR}: \delta 54.2,54.5,127.3$, 128.3, 129.2, 129.3, 134.5, 133.6, 140.2 and 161.1 ppm; LC-MS: ELSD pur. $93 \%$, UV pur. $100 \%$; $\mathrm{R}_{\mathrm{t}}$ $=8.88 \mathrm{~min} ; \mathrm{m} / \mathrm{z}: 236\left([\mathrm{M}+\mathrm{H}]^{+}\right)$.

3-(4-Phenyl-[1,2,3]triazol-1-yl)-propan-1-ol (1b2e): Prepared from $51 \quad \mathrm{mg} \quad(0.50 \mathrm{mmol})$ phenylacetylene and $56 \mathrm{mg}(0.55 \mathrm{mmol}) 3$-azidopropanol. The product was obtained as a white solid (102 mg, $99 \%$ \%. $\mathrm{C}_{12} \mathrm{H}_{15} \mathrm{~N}_{3} \mathrm{O}_{2}, \mathrm{M}=203.25 \mathrm{~g} \cdot \mathrm{mol}^{-1}$; mp: 90-92 ${ }^{\circ} \mathrm{C}$; FTIR: $v$ 3315, 3120, 2950, 2875, 1600 and $1052 \mathrm{~cm}^{-1}$; ${ }^{1} \mathrm{H}-\mathrm{NMR}: \delta 2.18(\mathrm{q}, J=6.0 \mathrm{~Hz}, 2 \mathrm{H}), 3.67(\mathrm{t}, J=6.0 \mathrm{~Hz}, 2 \mathrm{H}), 3.93(\mathrm{~s}, 1 \mathrm{H}), 4.62(\mathrm{t}$, $J=6.0 \mathrm{~Hz}, 2 \mathrm{H}), 7.59(\mathrm{~m}, 3 \mathrm{H}), 7.83(\mathrm{~m}, 2 \mathrm{H})$ and $7.84(\mathrm{~s}, 1 \mathrm{H}) ;{ }^{13} \mathrm{C}-\mathrm{NMR}: \delta 32.4,47.5,52.2,58.3,128.2$, 139.7 and 161.2 ppm; LC-MS: ELSD pur. $98 \%$, UV pur. $100 \% ; \mathrm{R}_{\mathrm{t}}=2.96 \mathrm{~min} ; \mathrm{m} / \mathrm{z}: 204\left([\mathrm{M}+\mathrm{H}]^{+}\right)$. 
(4-Phenyl-[1,2,3]triazol-1-yl)-acetic acid ethyl ester (1c2e): Prepared from $51 \mathrm{mg}$ (0.50 mmol) phenylacetylene and $71 \mathrm{mg}(0.55 \mathrm{mmol})$ ethyl azidoacetate. The product was obtained as a white solid (114 mg, 99 \%). $\mathrm{C}_{12} \mathrm{H}_{13} \mathrm{~N}_{3} \mathrm{O}_{2}, \mathrm{M}=231.26 \mathrm{~g} \cdot \mathrm{mol}^{-1}$; mp: 102-104 ${ }^{\circ} \mathrm{C}$; FTIR: v 3140, 3079, 3004, 2950, 1758, 1464, 1082 and $1044 \mathrm{~cm}^{-1}$; ${ }^{1} \mathrm{H}-\mathrm{NMR}: \delta 1.27(\mathrm{t}, J=7.2 \mathrm{~Hz}, 3 \mathrm{H}), 4.23$ (q, $\left.J=7.2 \mathrm{~Hz}, 2 \mathrm{H}\right), 5.13$ (s, $2 \mathrm{H}),, 7.35(\mathrm{~m}, 3 \mathrm{H}), 7.79(\mathrm{~m}, 2 \mathrm{H})$ and $7.89(\mathrm{~s}, 1 \mathrm{H}) ;{ }^{13} \mathrm{C}-\mathrm{NMR}: \delta 14.4,51.4,52.7,63.2,129.4,161.4$ and 166.0 ppm; LC-MS: ELSD pur. $99 \%$, UV pur. $100 \%$; $\mathrm{R}_{\mathrm{t}}=7.86 \mathrm{~min} ; \mathrm{m} / \mathrm{z}: 232\left([\mathrm{M}+\mathrm{H}]^{+}\right)$.

2,2,2-Trifluoro- $N$-[3-(4-phenyl-[1,2,3]triazol-1-yl)-propyl]-acetamide (1d2e): Prepared from $51 \mathrm{mg}$ $(0.50 \mathrm{mmol})$ phenylacetylene and $108 \mathrm{mg}(0.55 \mathrm{mmol}) \mathrm{N}$-(trifluoracetyl)-1-azido-3-aminopropane. The product was obtained as a white solid (128 mg, $86 \%$ ). $\mathrm{C}_{13} \mathrm{H}_{13} \mathrm{~F}_{3} \mathrm{~N}_{4} \mathrm{O}, \mathrm{M}=298.27 \mathrm{~g} \cdot \mathrm{mol}^{-1}$; mp: 158-160 ${ }^{\circ}$; FTIR: $v 3211,3045,2953,2896,1721,1193$, and $1144 \mathrm{~cm}^{-1} ;{ }^{1} \mathrm{H}-\mathrm{NMR}: \delta 2.25(\mathrm{~m}, 2 \mathrm{H})$, $3.43(\mathrm{t}, J=6.5 \mathrm{~Hz}, 2 \mathrm{H}), 4.50(\mathrm{t}, J=6.5 \mathrm{~Hz}, 2 \mathrm{H}), 7.05(\mathrm{~s}, 1 \mathrm{H}), 7.39(\mathrm{~m}, 4 \mathrm{H})$ and $7.82(\mathrm{~m}, 2 \mathrm{H}) \mathrm{ppm} ;{ }^{13} \mathrm{C}-$ NMR: $\delta$ 28.7, 36.6, 47.2, 117.7, 121.4, 125.1, 127.8, 128.8, 130.7, 146.2, 157.6 and 158.1 ppm; LCMS: ELSD pur. $96 \%$, UV pur. $100 \% ; \mathrm{R}_{\mathrm{t}}=7.88 \mathrm{~min} ; \mathrm{m} / \mathrm{z}: 299\left([\mathrm{M}+\mathrm{H}]^{+}\right)$.

1-Benzyl-4-trimethylsilanyl-1H-[1,2,3]-triazole (1a2f): Prepared from $49 \mathrm{mg}(0.50 \mathrm{mmol})$ (trimethylsilyl)acetylene and $50 \mathrm{mg}(0.375 \mathrm{mmol})$ benzyl azide. The product was obtained as a pale green solid (86 mg, $99 \%$ ). $\mathrm{C}_{12} \mathrm{H}_{17} \mathrm{~N}_{3} \mathrm{Si}, \mathrm{M}=231.38 \mathrm{~g} \cdot \mathrm{mol}^{-1}$; mp: $74-76^{\circ} \mathrm{C}$; FTIR: $v 3286,3115,3061$, 2953, 2920, 1674, 1542, 1438, 1318, 1280 and $1168 \mathrm{~cm}^{-1} ;{ }^{1} \mathrm{H}-\mathrm{NMR}: \delta 0.11$ (s, 9H), 5.56 (s, 2H), 7.24$7.34(\mathrm{~m}, 5 \mathrm{H}), 7.26(\mathrm{~s}, 1 \mathrm{H}) \mathrm{ppm} ;{ }^{13} \mathrm{C}-\mathrm{NMR}: \delta 0.02,54.6,129.2,129.4,130.2$ and $136.1 \mathrm{ppm}$; LC-MS: ELSD pur. $96 \%$, UV pur. $100 \% ; \mathrm{R}_{\mathrm{t}}=11.23 \mathrm{~min} ; \mathrm{m} / \mathrm{z}: 232\left([\mathrm{M}+\mathrm{H}]^{+}\right)$.

3-(4-(trimethylsilyl)-1H-1,2,3triazol-1-yl)propan-1-ol (1b2f): Prepared from $49 \mathrm{mg}$ (0.50 mmol) (trimethylsilyl)acetylene and $38 \mathrm{mg}(0.375 \mathrm{mmol}) 3$-azidopropanol. The product was obtained as a pale green solid (54 mg, 72\%). $\mathrm{C}_{8} \mathrm{H}_{17} \mathrm{~N}_{3} \mathrm{OSi}, \mathrm{M}=199.33 \mathrm{~g} \cdot \mathrm{mol}^{-1}$; mp :58-60 ${ }^{\circ} \mathrm{C}$; FTIR: $v 3290,2920$, 2850, 1650, 1461, 1213,1172 and $1049 \mathrm{~cm}^{-1}$; ${ }^{1} \mathrm{H}-\mathrm{NMR}: \delta 0.12$ (s,9H), 1.94 (q, $\left.J=2 \mathrm{~Hz}, 2 \mathrm{H}\right), 3.44-3.47$ $(\mathrm{m}, 2 \mathrm{H}), 3.55-3.58(\mathrm{t}, J=4.5 \mathrm{~Hz}, 1 \mathrm{H}), 4.34-4.38(\mathrm{t}, J=6 \mathrm{~Hz}, 2 \mathrm{H})$ and $7.41(\mathrm{~s}, 1 \mathrm{H}) \mathrm{ppm} ;{ }^{13} \mathrm{C}-\mathrm{NMR}: \delta 0.02$, 34.1, 47.6, 59.7 and 130.7 ppm; LC-MS: ELSD pur. $90 \%$, UV pur. $100 \%$; $\mathrm{R}_{\mathrm{t}}=2.74 \mathrm{~min} ; \mathrm{m} / \mathrm{z}: 200$ $\left([\mathrm{M}+\mathrm{H}]^{+}\right)$.

Ethyl 2-(4-(trimethylsilyl)-1H-1,2,3-triazol-1-yl)acetate (1c2f): Prepared from $49 \mathrm{mg}$ (0.50 mmol) (trimethylsilyl)acetylene and $48 \mathrm{mg}(0.375 \mathrm{mmol})$ ethyl azidoacetate. The product was obtained as a brown oil (70 mg, $83 \%) . \mathrm{C}_{9} \mathrm{H}_{17} \mathrm{~N}_{3} \mathrm{O}_{2} \mathrm{Si}, \mathrm{M}=227.34 \mathrm{~g} \cdot \mathrm{mol}^{-1}$; FTIR: $v 2928,2854,1746,1455,1372$, 1213 and $1023 \mathrm{~cm}^{-1}$; ${ }^{1} \mathrm{H}-\mathrm{NMR}$ : $\delta 0.12$ (s, 9H), 1.12 (t, $\left.J=8 \mathrm{~Hz}, 3 \mathrm{H}\right), 4.07-4.09$ (q, $\left.J=1 \mathrm{~Hz}, 2 \mathrm{H}\right), 5.01$ (s, $1 \mathrm{H})$ and $7.49(\mathrm{~s}, 1 \mathrm{H}) \mathrm{ppm} ;{ }^{13} \mathrm{C}-\mathrm{NMR}: \delta 0.02,15.2,51.4,63.5,131.5$ and $167.7 \mathrm{ppm}$; LC-MS: ELSD pur. $99 \%$, UV pur. $100 \% ; \mathrm{R}_{\mathrm{t}}=8.50 \mathrm{~min} ; \mathrm{m} / \mathrm{z}: 228\left([\mathrm{M}+\mathrm{H}]^{+}\right)$.

2,2,2-trifluoro-N-(3-(4-trimethylsilyl)-1H-1,2,3triazole-1-yl)propyl)acetamide (1d2f): Prepared from $46 \mathrm{mg}(0.50 \mathrm{mmol})$ (trimethylsilyl)acetylene and $59 \mathrm{mg}(0.375 \mathrm{mmol}) \mathrm{N}$-(trifluoracetyl)-1-azido-3aminopropane. The product was obtained as a pale green solid (101 mg, $90 \%)$. $\mathrm{C}_{10} \mathrm{H}_{17} \mathrm{~F}_{3} \mathrm{~N}_{4} \mathrm{OSi}, \mathrm{M}=$ $294.35 \mathrm{~g} \cdot \mathrm{mol}^{-1}$; mp: $120-122^{\circ} \mathrm{C}$; FTIR: v3186, 3124, 3073, 2962, 1721, 1571, 1185 and $1156 \mathrm{~cm}^{-1}$; 
${ }^{1} \mathrm{H}-\mathrm{NMR}\left(\mathrm{CD}_{3} \mathrm{CN}\right): \delta 0.15(\mathrm{~s}, 9 \mathrm{H}), 1.82(\mathrm{t}, J=3 \mathrm{~Hz}, 2 \mathrm{H}), 3.15-3.17(\mathrm{~m}, 2 \mathrm{H}), 4.26-4.30(\mathrm{q}, J=3 \mathrm{~Hz}, 2 \mathrm{H})$, 7.49-7.50 (m, $1 \mathrm{H})$ and $7.68(\mathrm{~s}, 1 \mathrm{H}) \mathrm{ppm} ;{ }^{13} \mathrm{C}-\mathrm{NMR}\left(\mathrm{CD}_{3} \mathrm{CN}\right): \delta 0.02,28.7,36.5,47.7,132.6$ and 157.7 ppm; LC-MS: ELSD pur. $99 \%$, UV pur. 100\%; $\mathrm{R}_{\mathrm{t}}=10.15 \mathrm{~min} ; \mathrm{m} / z: 335\left([\mathrm{M}+\mathrm{MeCN}]^{+}\right)$.

\section{Acknowledgements}

This work was made possible with the help of grants CNRS 8151, INSERM U640 and SESAME Program from Ile-de-France. This is a part of the work of I. J. for an international Ph.D. Program between Université de Paris (UPMC) - France and Université du 7 novembre de Carthage (Faculté de Bizerte) - Tunisia. A Ph. D. fellowship from Tunisia to I. J. is greatly acknowledged.

\section{References and Notes}

1. Kolb, H.C.; Finn, M.G.; Sharpless, K.B. Click Chemistry: Diverse Chemical Function from a Few Good Reactions. Angew. Chem. 2001, 113, 2056-2075; Angew. Chem. Int. Ed. 2001, 40, $2004-$ 2021.

2. Kolb, H.C.; Sharpless, K.B. The growing impact of click chemistry on drug discovery. Drug Discov. Today 2003, 8, 1128-1137.

3. Moses, J.E.; Moorhouse, A.D. The growing applications of click chemistry. Chem. Soc. Rev. 2007, 36, 1249-1262.

4. Huisgen, R. 1,3-Dipolar Cycloadditions. Past and Future. Angew. Chem. 1963, 75, 604-637; Angew. Chem., Int. Ed. Engl. 1963, 2, 565-598.

5. Tornøe, C.W.; Meldal, M. Peptidotriazoles: Copper(I)-Catalyzed 1,3-Dipolar Cycloadditions on Solid phase. In 17th American Peptides Symposium Proceedings Book. Peptides: The Wave of the Future; Lebl, M., Houghten, R.A., Eds.; American Peptide Society and Kluwer Academic: San Diego, USA, 2001; pp 263-264.

6. Tornøe, C.W.; Christensen, C.; Meldal, M. Peptidotriazoles on Solid Phase: [1,2,3]-Triazoles by Regiospecific Copper(I)-Catalyzed 1,3-Dipolar Cycloadditions of Terminal Alkynes to Azides. $J$. Org. Chem. 2002, 67, 3057-3064.

7. Rostovtsev, V.V.; Green, L.G.; Fokin, V.V.; Sharpless, K.B. A Stepwise Huisgen Cycloaddition Process: Copper(I)-Catalyzed Regioselective Ligation of Azides and Terminal Alkynes. Angew. Chem. 2002, 114, 2708-2711; Angew. Chem. Int. Ed. 2002, 41, 2596-2599.

8. For reviews see: Bock, V. D.; Hiemstra, H.; van Maarseveen, J. H. Cu-Catalyzed Alkyne-Azide Click Cycloadditions from a Mechanistic and Synthetic Perspective. Eur. J. Org. Chem. 2006, 5168 and references [9-10].

9. Wu, P.; Fokin, V.V. Catalytic Azide-Alkyne Cycloaddition: Reactivity and Applications. Aldrichim. Acta 2007, 40, 7-17.

10. Meldal M.; Tornøe, C.W. Cu-Catalyzed Azide-Alkyne Cycloaddition. Chem. Rev. 2008, 108, 2952-3015.

11. For some selected examples, see: Zhang, X.; Hsung, R.P.; Li, H. A triazole-templated ringclosing metathesis for constructing novel fused and bridged triazoles. Chem. Commun. 2007, 2420-2422 and references [12-13]. 
12. Bertrand, P.; Gesson, J.-P. Click Chemistry with $O$-Dimethylpropargylcarbamate for Preparation of pH-Sensitive Functional Groups. A Case Study. J. Org. Chem. 2007, 72, 3596-3599.

13. Peddibhotla, S.; Dang, Y.; Liu, J.O.; Romo, D. Simultaneous Arming and Structure/Activity Studies of Natural Products Employing O-H Insertions: An Expedient and Versatile Strategy for Natural Products-Based Chemical Genetics. J. Am. Chem. Soc. 2007, 129, 12222-12231.

14. For some selected examples, see: Beckmann, H.S.G.; Wittmann, V. One-Pot Procedure for Diazo Transfer and Azide-Alkyne Cycloaddition: Triazole Linkages from Amines. Org. Lett. 2007, 9, 1-4 and references [15-16].

15. Tao, C.-Z.; Cui, X.; Li, J.; Liu, A.-X.; Liu, L.; Guo, Q.-X. Copper-catalyzed synthesis of aryl azides and 1-aryl-1,2,3-triazoles from boronic acids. Tetrahedron Lett. 2007, 48, 3525-3529.

16. Barral, K.; Moorhouse, A.D.; Moses, J.E. Efficient Conversion of Aromatic Amines into Azides: A One-Pot Synthesis of Triazole Linkages. Org. Lett. 2007, 9, 1809-1811.

17. Chassaing, S.; Kumarraja, M.; Sani Souna Sido, A.; Pale, P.; Sommer, J. Click Chemistry in Cu zeolites: The Huisgen [3 + 2]-Cycloaddition. Org. Lett. 2007, 9, 883-886.

18. Lipshutz, B.H.; Taft, B.R. Heterogeneous Copper-in-Charcoal-Catalyzed Click Chemistry. Angew. Chem. 2006, 118, 8415-8418; Angew. Chem., Int. Ed. 2006, 45, 8235-8238.

19. Jlalia, I.; Elamari, H., Meganem, F.; Herscovici, J.; Girard, C. Copper(I)-doped Wyoming's montmorillonite for the synthesis of disubstituted 1,2,3-triazoles. Tetrahedron Lett. 2008, 49, 6756-6758.

20. For some selected examples, see: Appukkuttan, P.; Dehaen, W.; Fokin, V.V.; Van der Eycken, E. A Microwave-Assisted Click Chemistry Synthesis of 1,4-Disubstituted 1,2,3-Triazoles via a Copper(I)-Catalyzed Three-Component Reaction. Org. Lett. 2004, 6, 4223-4225 and references [21-22] therein.

21. Himo, F.; Lovell, T.; Hilgraf, R.; Rostovtsev, V.V.; Noodleman, L.; Sharpless, K.B.; Fokin, V.V. Copper(I)-Catalyzed Synthesis of Azoles. DFT Study Predicts Unprecedented Reactivity and Intermediates. J. Am. Chem. Soc. 2005, 127, 210-216.

22. David, O.; Maisonneuve, S.; Xie, J. Generation of new fluorophore by Click chemistry: synthesis and properties of $\beta$-cyclodextrin substituted by 2-pyridyl triazole. Tetrahedron Lett. 2007, 48, 6527-6530.

23. Durán Pachón, L.; van Maarseveen, J.H.; Rothenberg, G. Click Chemistry: Copper Clusters Catalyse the Cycloaddition of Azides with Terminal Alkynes. Adv. Synth. Catal. 2005, 347, 811815.

24. Molteni, G.; Bianchi, C.L.; Marinoni, G.; Santo, N.; Ponti, A. Cu/Cu-oxide nanoparticles as catalyst in the click azide-alkyne cycloaddition. New J. Chem. 2006, 30, 1137-1139.

25. Girard, C.; Önen, E.; Aufort, M.; Beauvière, S.; Samson, E.; Herscovici, J. Reusable PolymerSupported Catalyst for the [3+2] Huisgen Cycloaddition in Automation Protocols. Org. Lett. 2006, $8,1689-1692$.

26. For another exemple of a polystyrene-based supported catalyst, see: Chan, T.R.; Fokin, V.V. Polymer-Supported Copper(I) Catalysts for the Experimentally Simplified Azide-Alkyne Cycloaddition. QSAR Comb. Sci. 2007, 26, 1274-1279.

27. Walsh, P.J.; Li, H.; Anaya de Parrodi, C. A Green Chemistry Approach to Asymmetric Catalysis: Solvent-Free and Highly Concentrated Reactions. Chem. Rev. 2007, 107, 2503-2545. 
28. During this work, manuscript preparation and submissions, other examples using solvent-free conditions in the Huisgen reaction were published, see: Díez-González, S.; Correa, A.; Cavallo, L; Nolan, S.P. (NHC)Copper(I)-Catalyzed [3 + 2] Cycloaddition of Azides and Mono- or Disubstituted Alkynes. Chem. Eur. J. 2006, 12, 7558-7564 and references [29-31] therein.

29. Guezguez, R.; Bougrin, K.; El Akri, K.; Benhida, R. A highly efficient microwave-assisted solvent-free synthesis of $\alpha$ - and $\beta$-2'-deoxy-1,2,3-triazolyl-nucleosides. Tetrahedron Lett. 2006, 47, 4807-4811.

30. El Akri, K.; Bougrin, K.; Balzarini, J.; Faraj, A.; Benhida, R. Efficient synthesis and in vitro cytostatic activity of 4-substituted triazolyl-nucleosides. Bioorg. Med. Chem. Lett. 2007, 17, 6656-6659.

31. Li, P.; Wang, L.; Zhang, Y. $\mathrm{SiO} 2-\mathrm{NHC}-\mathrm{Cu}(\mathrm{I})$ : an efficient and reusable catalyst for [3+2] cycloaddition of organic azides and terminal alkynes under solvent-free reaction conditions at room temperature. Tetrahedron 2008, 64, 10825-10830.

32. Smith, C.D.; Baxendale, I.R.; Lanners, S.; Hayward, J.J.; Smith, S.C.; Ley, S.V. [3 + 2] Cycloaddition of acetylenes with azides to give 1,4-disubstituted 1,2,3-triazoles in a modular flow reactor. Org. Biomol. Chem. 2007, 5, 1559-1561.

33. For an example, see: Katritzky, A.R.; Singh, S.K. Synthesis of C-Carbamoyl-1,2,3-triazoles by Microwave-Induced 1,3-Dipolar Cycloaddition of Organic Azides to Acetylenic Amides. J. Org. Chem. 2002, 67, 9077-9079.

34. Pourcelot, G.; Cadiot, P. Preparation of propargylic, allenic and acetylenic derivatives of the elements of Group VIB. Bull. Soc. Chim. Fr. 1966, 3016-3024.

35. Alvarez, S.G.; Alvarez, M.T. A Practical Procedure for the Synthesis of Alkyl Azides at Ambient Temperature in Dimethyl Sulfoxide in High Purity and Yield. Synthesis 1997, 413-414.

36. Hooper, N.; Beeching, L.J.; Dyke, J.M.; Morris, A.; Ogden, J.S.; Dias, A.A.; Costa, M.L.; Barros, M.T.; Cabrell, M.H.; Moutinho, A.M.C. A Study of the Thermal Decomposition of 2Azidoethanol and 2-Azidoethylacetate by Ultraviolet PES and Matrix Isolation Spectroscopy. $J$. Phys. Chem. A. 2002, 106, 9968-9975.

37. Scheel, A.J.; Komber, H.; Voit, B.I. Novel Hyperbranched Poly([1,2,3]-triazole)s Derived from $\mathrm{AB}_{2}$ Monomers by a 1,3-Dipolar Cycloaddition. Macromol. Rapid Commun. 2004, 25, $1175-$ 1180.

38. Carboni, B.; Benalil, A.; Vaultier, M. Aliphatic amino azides as key building blocks for efficient polyamine syntheses. J. Org. Chem. 1993, 58, 3736-3741.

Sample Availability: Samples of the compounds are available from the authors.

(C) 2009 by the authors; licensee Molecular Diversity Preservation International, Basel, Switzerland. This article is an open-access article distributed under the terms and conditions of the Creative Commons Attribution license (http://creativecommons.org/licenses/by/3.0/). 